So Young Park Employee of: Eli Lilly and Company, Matthew Hufford Shareholder of: Eli Lilly and Company, Employee of: Eli Lilly and Company, Peter Nash Grant/research support from: AbbVie, Bristol-Myers Squibb, Eli Lilly, Janssen, Novartis, Pfizer Inc, Roche, Sanofi, UCB, Consultant for: AbbVie, Bristol-Myers Squibb, Eli Lilly, Janssen, Novartis, Pfizer Inc, Roche, Sanofi, UCB, Speakers bureau: AbbVie, Bristol-Myers Squibb, Eli Lilly, Janssen, Novartis, Pfizer Inc, Roche, Sanofi, UCB DOI: 10.1136/annrheumdis-2019-eular.1240

\section{SAT0375 MILD COGNITIVE IMPAIRMENTIN PSORIATIC ARTHRITIS: PREVALENCE AND ASSOCIATED FACTORS}

Marco Di Carlo ${ }^{1,1}$, Andrea Becciolini ${ }^{2}$, Antonella Incorvaia ${ }^{1}$, Giacomo Beci ${ }^{1}$, Martina Biggioggero ${ }^{2}$, Ennio Giulio Favalli ${ }^{2}$, Fausto Salaffi ${ }^{1}{ }^{1}$ Rheumatological Clinic, Dipartimento di Scienze Cliniche e Molecolari - Università Politecnica delle

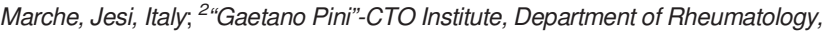
Milan, Italy

Background: A growing body of data demonstrated that systemic inflammation is a predisposing condition for developing cognitive impairment Different studies highlighted that psoriasis ( $\mathrm{PsO}$ ), a multisystem chronic inflammatory disorder, is associated with cognitive impairment. No data are available regarding psoriatic arthritis (PsA).

Objectives: To assess the prevalence and the factors associated with mild cognitive impairment $(\mathrm{MCl})$ in patiens suffering from PsA.

Methods: Consecutive PsA patients, classified as suffering from PsA with the CIASsification criteria for Psoriatic ARthritis (CASPAR), were enrolled in this study. For each patient were collected the sociodemographic data (age, gender, years of school attendance, years of articular disease duration, years of cutaneous disease duration, treatment), the values of C-reactive protein (CRP) and the following clinical variables: 68 tender joint count (TJC), 66 swollen joint count (TJC), the Leeds Enthesitis Index (LEI), the dactylitis digit count, the Psoriasis Area and Severity Index (PASI), $10 \mathrm{~cm}$ numerical rating scale (NRS) for pain and for assessment of disease activity (PaGA), the Dermatology Life Quality Index (DLQI), the 12-item Psoriatic Arthritis Impact of Disease (PsAID12), the Health Assessment Questionnaire (HAQ), and the Self-admistered Comorbidity Questionnaire (SCQ). Cognitive impairment was assessed through the Montreal Cognitive Assessment (MoCA). The exclusion criteria were represented by the presence of: age older than 70 years, major depressive disorder, dementia, Parkinson's disease, active skin disease other than PsO, concomitant inflammatory joint conditions (such as gout or calcium pyrophosphate deposition), and comorbid fibromyalgia.

Results: The study involved 96 PsA patients, with a mean age of 52.8 years. Twenty $(20.8 \%)$ patients were suffering from PsA sine PsO. The mean articular disease duration was 9.9 years, and the mean cutaneous disease duration was 14.9 years. The mean Disease Activity index for Psoriatic Arthritis (DAPSA) was 11.8 , and the mean PASI was $0.95 . \mathrm{MCl}$ (defined as a MoCA score <26/30) was detected in 47 (48.9\%) patients, with a mean value of MoCA of $25.1 \pm 3.1$. Short-term memory was the most affected domain (mean value 2.6 on 0-5 point scale). The variables under investigation significantly correlated with a worsening cognitive performance (Spearman's Rho) were functional capacity (MoCA vs HAQ, $r_{s}$ $=-0.227, p=0.026$ ) and fatigue (MoCA vs PsAID item 2, $r_{s}=-0.222$, $\mathrm{p}=0.029$ ).

Conclusion: $\mathrm{MCl}$ is present in a significant proportion of PsA patients, and is present in a relative young age. $\mathrm{MCl}$ occurrence is associated with the presence of a worse functional capacity and a greater fatigue. Short-term memory is the more frequently affected domain. Although not predominantly of rheumatological interest, the $\mathrm{MCl}$ presence should be carried out in all patients with PsA.

\section{REFERENCES}

[1] Gisondi P, Sala F, Alessandrini F, et al. Mild cognitive impairment in patients with moderate to severe chronic plaque psoriasis. Dermatology. 2014;228(1):78-85.

[2] Colgecen E, Celikbilek A, Keskin DT. Cognitive Impairment in Patients with Psoriasis: A Cross-Sectional Study Using the Montreal Cognitive Assessment. Am J Clin Dermatol. 2016;17(4):413-9.
Disclosure of Interests: Marco Di Carlo: None declared, Andrea Becciolini: None declared, Antonella Incorvaia: None declared, giacomo beci: None declared, Martina Biggioggero: None declared, Ennio Giulio Favalli: None declared, Fausto Salaffi Grant/research support from: Abbvie, Roche, Novartis, BMS, Pfizer, Sanofi, Speakers bureau: Abbvie, Roche, Novartis, Pfizer, Sanofi, BMS

DOI: 10.1136/annrheumdis-2019-eular.4695

\section{SAT0376 MAKING THE NEXT STEPS IN PSORIATIC ARTHRITIS MANAGEMENT: DOMAIN DRIVEN, TREAT-TO-TARGET, TAILORED MANAGEMENT APPROACH}

Yasser El Miedany ${ }^{1}$, Mohammed Hassan Abu-Zaid ${ }^{2}$, Waleed Hassan ${ }^{3}$, Mohammed A. Mortada ${ }^{4}$, Dalia Mekkawy ${ }^{5}$, Samah Ismail Nasef ${ }^{6}$, Nadia El Aroussy ${ }^{5}$, Mervat Eissa ${ }^{7}$, Yossra Atef $^{8}$, Eiman Dessouki ${ }^{9}$, Maha El Gaafary ${ }^{10}$. ${ }^{1}$ Darent Valley Hospital, Rheumatology, Dartford, United Kingdom; ${ }^{2}$ Tanta University School of Medicine, Rheumatology and Rehabilitation, Tanta, Egypt, ${ }^{3}$ Benha University school of medicine, Rheumatology and Rehabilitation, Benha, Egypt, ${ }^{4}$ Zagazig University school of medicine, Rheumatology and Rehabilitation, Zagazig, Egypt, ${ }^{5}$ Ain Shams University School of medicine, Rheumatology and Rehabilitation, Cairo, Egypt, ${ }^{6}$ Suez canal university, Rheumatology and Rehabilitation, Ismailia, Egypt, ${ }^{7}$ Cairo University school of medicine, Rheumatology and Rehabilitation, Cairo, Egypt, ${ }^{8}$ Assiut University, school of medicine,

Rheumatology and Rehabilitation, Assiut, Egypt, ${ }^{9}$ Alexandria University, school of medicine, Opthalmology, Alexandria, Egypt, ${ }^{10}$ Ain Shams University school of medicine, Community and Public Health, Cairo, Egypt

Background: The diverse clinical picture of psoriatic arthritis (PsA) sug gests the need to identify suitable therapies to address the different combinations of clinical manifestations and comorbidities. The current treatment paradigms recommend early diagnosis and treatment, and a strategic, target orientated approach, aiming at a low disease activity status. The introduction of new treatment modalities highlighted the need for guidelines to prioritize these options for doctors and patients

Objectives: To develop an evidence-based recommendation for the management and treatment of PsA. The management paradigm should cover all domains of psoriatic disease and provide a stepwise tailored treatment options giving clear advice on treatment from the initial diagnosis, including inclusion/exclusion criteria for treatment, monitoring requirements and how to quantify response to treatment.

Methods: A Steering Committee formulated a set of overarching principles for the management of PsA based on evidence derived from a systematic literature review. These were subsequently discussed, amended and voted on by a Task Force of 20 rheumatologists, dermatologists and patient research partners. Using the nominal group technique and Delphi method, 7domains were identified (peripheral arthritis, axial disease, enthesitis, dactylitis, skin and nails as well as comorbidities including uveitis). A multidisciplinary, evidence- and consensusbased treatment recommendations for PSA were developed for each of the domains based on three consensus discussions. A set of recommendations for a sequential DMARD/biologic treatment algorithm for patients with PsA was also set.

Results: literature review provided evidence regarding an optimised domain specific, treat-to- target approach to management. The guidelines addressed both drug and non-drug interventions. 20-statements regarding diagnosis, disease activity scoring, US/MRI scanning, and drug therapy were generated. Percentage of positive votes ranged between $86-100 \%$; whereas mean \pm SD level of agreement was $9.6+0.3$ The main treatment outcome is to achieve remission status or at least low disease activity state, which should be based on a shared decision with parents/patients. Treatment should be adapted according to disease activity. Treatment should be also adapted if the remission state got lost.

Conclusion: Although no clear correlation exists between joint inflammation and the skin in every patient, the skin and joint aspects of the disease often must be treated simultaneously. Treatment recommendations for the cardinal physical manifestations of PsA were developed based on a literature review and consensus between rheumatologists, dermatologists and patients. It is anticipated that periodic updates will take place using this framework as new data become available.

Disclosure of Interests: None declared

DOI: 10.1136/annrheumdis-2019-eular.1850 